\title{
Development of a gaseous recoil-proton detector for neutron flux measurements between 0.2 and $2 \mathrm{MeV}$ neutron energy
}

\author{
P. Marini ${ }^{\mathrm{a}, \mathrm{b}}$, L. Mathieu, M. Aiche, T. Cheron, P. Hellmuth, J.L. Pedroza, S. Czajkowski, B. Jurado, \\ and I. Tsekhanovich
}

CENBG, CNRS/IN2P3-Université de Bordeaux, Chemin du Solarium, BP. 120, 33175 Gradignan, France

\begin{abstract}
Absolute measurements of neutron fluence are an essential prerequisite of neutron-induced cross section measurements, neutron beam lines characterisation and dosimetric investigations. Precise neutron flux measurements can be performed with respect to the $\mathrm{H}(\mathrm{n}, \mathrm{p})$ elastic cross section. The use of this technique, with silicon proton recoil detectors, is not straightforward below incident neutron energy of $1 \mathrm{MeV}$, due to a high background in the detected proton spectrum. Experiments carried out at the AIFIRA facility identified its origin. Based on these investigations, a gaseous recoil-proton detector has been designed, with a reduced low energy background. Preliminary results of the first tests of the developed detector are discussed here.
\end{abstract}

\section{Introduction}

The development of Generation IV nuclear systems relies on accurate simulation based on high quality nuclear data [1]. However, despite the experimental efforts, large discrepancies and uncertainties exist nowadays on data in the actinide region [2]. In particular, a precise and accurate knowledge of fission cross sections is of primary important for determining the nuclear core behaviour or waste incineration capabilities. At the energies where a fast reactor neutron flux is peaked (about $100 \mathrm{keV} \mathrm{[3]),}$ the non-fissile isotope fission cross sections are quite low, however some of them (for instance ${ }^{240-242} \mathrm{Pu}(\mathrm{n}, \mathrm{f})$ ) are not negligible and may have an impact on the waste production. Fission cross section measurements have therefore to be improved in the $0.1-1 \mathrm{MeV}$ energy range [4].

A cross section measurement by direct neutron irradiation of the sample of interest requires the precise knowledge of the neutron flux impinging on the sample. Typically, the neutron flux is determined with respect to well-known fission standard reactions $\left({ }^{235} \mathrm{U}(\mathrm{n}, \mathrm{f}),{ }^{238} \mathrm{U}(\mathrm{n}, \mathrm{f})\right.$, $\left.{ }^{237} \mathrm{~Np}(\mathrm{n}, \mathrm{f})\right)$. However, this introduces a strong correlation between independent measurements based on the same standard. Independent measurements can be carried out with respect to the $H(n, p)$ elastic cross section. In this method, the neutron flux is converted into a proton flux, by mean of a $\mathrm{H}$-rich foil. The charged protons can be easily detected, for instance, in a silicon detector. Different types of recoil-proton detector exist, depending on the type of experiment, the facility environment, the neutron energy range and the target accuracy of the measurement [5-15].

\footnotetext{
a e-mail: paola.marini@cea.fr

${ }^{\mathrm{b}}$ Present address: CEA, DAM, DIF, 91297 Arpajon, France
}

\section{Silicon-based recoil-proton detector}

The recoil-proton detector developed by the ACEN group of the CENBG institute, for measurements in the MeV energy range, consists of a polypropylene foil (4 to $50 \mu \mathrm{m}$ ), a $50 \mu \mathrm{m}$ silicon detector, or a silicon detectors telescope, and a collimator to well-define the covered solid angle. The protons detected in the silicon have kinetic energies close to the incident neutron energy, thus producing a proton peak in the proton spectrum, with a very good signalto-background ratio. The integral of the proton peak is then used to deduce the incident neutron flux. A detailed description of the method and of the detector itself can be found in $[10,16]$. Previous studies showed that, while in measurements at $2 \mathrm{MeV}$ and $5 \mathrm{MeV}$ uncertainties of the order of $1 \%$ could be achieved in the proton peak integral [10], this was not the case at and below $1 \mathrm{MeV}(30 \%$ at $700 \mathrm{keV}$ ) [16], where a strong component of background significantly reduced the signal-to-background ratio. Investigations performed subsequently at the AIFIRA facilities, combined with MCNP simulations, showed that the background arises from Compton scattered electrons, generated by $\gamma$-rays produced by the neutron source and/or neutron capture. Contrary to protons, whose range is small compared to the detector thickness (down to $2 \mu \mathrm{m}$ for $0.2 \mathrm{MeV}$ proton), electrons deposit their energy, although with a smaller stopping power, in the whole detector thickness $(2800 \mu \mathrm{m}$ range for $1 \mathrm{MeV}$ electron), generating a significant background. Thinner silicon detectors, with thickness of few $\mu \mathrm{m}$, would reduce the electron contribution. However, the recoil-proton detector would not be flexible with respect to the neutron energy, besides being quite expensive. A possible solution is the use of a gaseous detector. A proportional counter protonrecoil spectrometer filled with hydrogen and allowing neutron $/ \gamma$ (electrons) discrimination was developed in the 60 's by Bennett et al. [5]. The comparison to this detector of the newly developed gas detector is discussed in the 


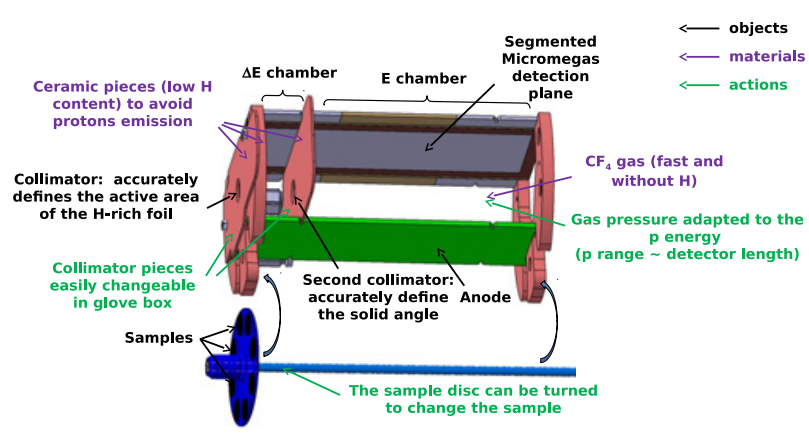

Figure 1. Schema of the detector. The sample disk, positioned in front of the first collimator (see text), is shown separately for clarity purpose.

following, despite the different scopes of the two detectors (neutron energy spectra vs neutron flux measurements).

\section{A gas recoil-proton detector prototype}

The ACEN group has developed a gaseous recoilproton detector with the aim of fulfilling the following requirements: the detector i) provides a precise neutron flux measurement; ii) is adapted to work in high neutron density environments and with highly radioactive samples; iii) is adapted for a neutron energy range from 0.2 to $2 \mathrm{MeV}$; iv) has a low sensitivity to electrons; and v) allows for a direct vs. scattered neutron discrimination. A scheme of the detector is shown in Fig. 1.

The detector is constituted by a H-rich foil and a segmented $\Delta \mathrm{E}-\mathrm{E}$ ionization chamber $\left(4 \times 4 \times 12 \mathrm{~cm}^{3}\right)$. The chamber is filled with $\mathrm{CF}_{4}$ gas at an adjustable pressure, from few tens of mbar to slightly above 1 bar, to adapt the proton (or $\alpha$ ) range to the detector length. This allows to reduce as much as possible the detector sensitivity to Compton electrons. The coincidence between the $\Delta \mathrm{E}$ and $\mathrm{E}$ sides of the ionization chamber allow a first discrimination between direct and scattered neutrons, not possible in Bennett's detector since protons are created in the whole gas volume.

Eight different $\mathrm{H}$ deposit (from 1 to $10 \mu \mathrm{m}$ thick) can be mounted on a turning disk. The thinnest samples will be evaporated tristearine, while the thickest ones are polypropylene foils. An empty hole is available for background measurements. The possibility to select the sample thickness adapted to the neutron incident energy is crucial to obtain precise measurements at low neutron energy since it allows to keep the proton energy loss in the $\mathrm{H}$-foil below $10 \%$ and thus to reduce their energy spread. Moreover we stress here the need of precisely knowing the quantity of $\mathrm{H}$ atoms to determine the neutron flux. This is a major difference with Bennett's detector, where the $\mathrm{H}$ quantity can not be precisely controlled.

The detection plane is a Micromegas, segmented in 64 pads, as shown in Fig. 2a. The Micromegas allows to multiply the number of primary electrons generated by the proton ionization of the gas by a factor about $10^{6}$. Indeed, the number of primary electrons can be as low as 1000 electrons per chamber side for $200 \mathrm{keV}$ neutron energy. The detection plane segmentation allows both to reduce the electronic noise associated to the pad capacitance, and to

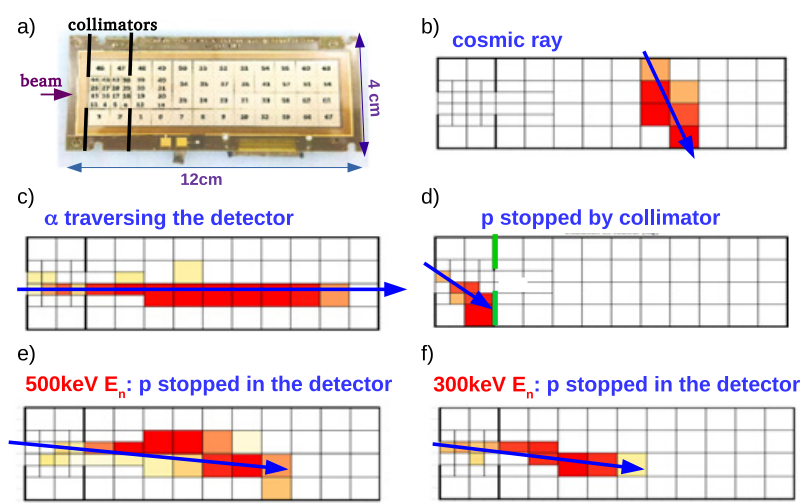

Figure 2. a) Detection plane segmentation b-f) examples of reconstructed trajectories.

perform a raw track analysis, to reject false coincidences with abnormal trajectories.

Two ceramic collimators, one placed right after the sample, the other between the two ionization chambers, provide a well-defined geometry, essential for precise neutron flux measurements. Collimators with different diameters, depending on the neutron energy, can be easily replaced, even in a glove box.

Finally, the gas and the ceramic materials have been chosen for their very low or absent hydrogen content, so that no additional protons can be emitted by the gas or the detector structure.

A more detailed description of the detector, as well as of the associated electronics, based on the GET system [17], will be presented in a forthcoming publication.

\subsection{Performances: Preliminary experimental results}

The detector prototype was delivered at the end of June 2016 and a first experiment to evaluate its performances was carried out in July 2016 at the AIFIRA facility.

Measurements were performed with quasi-monoenergetic neutron beam, with energies from 1 to $0.3 \mathrm{MeV}$, and with a $3 \alpha$ source. The gas pressure was varied from 100 to 30 mbar, depending on the neutron energy, and the applied voltage was adapted consequently. The detector was placed in an aluminium chamber, filled with gas, that reduces as much as possible gas pressure fluctuations. Measurements were performed to:

- Evaluate the direct vs scattered neutron discrimination. Any neutron interacting with matter $(\mathrm{H}$ from the target deposit or surrounding materials) may generate protons which will be detected. It is therefore crucial to discriminate protons coming from the direct interaction of the neutron beam with the deposited $\mathrm{H}$ (direct neutrons) from those coming from the interaction of the beam with any other material than the $\mathrm{H}$ deposit (scattered neutrons). Direct and scattered neutrons could be discriminated imposing a $\Delta \mathrm{E}-\mathrm{E}$ coincidence. Also, the trajectory reconstruction (Fig. 2b-f) places further constraints on the direct neutron selection. On these basis, we can remove protons scattered at high angles (Fig. 2d), protons generated by scattered neutrons, protons generated elsewhere than in the $\mathrm{H}$ foil. 


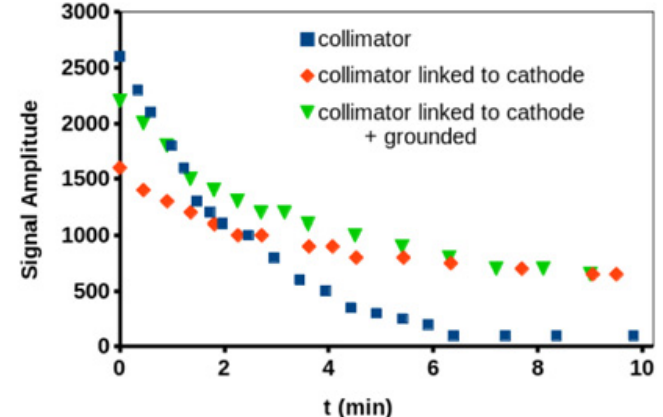

Figure 3. Evolution of the signal amplitude (a.u.) of the first row of pads with irradiation time.

- Study the detector sensitivity to: i) $\mathbf{e}^{-}$and/or $\gamma$-rays: no signal associated to electrons and $\gamma$-rays were detected; ii) cosmic rays: easily discriminated based on the reconstructed trajectory (Fig. 2b); iii) a direct neutron or $\alpha$ irradiation of the Micromegas: no signals associated to these events were detected.

- Evaluate the possibility of a determining a relative calibration of each detection pad. While the electronic gain can be easily determined by injecting a pulser in the electronic chain, the gain of each pad is strongly dependent on the gas pressure, as well as on the local Micromegas structure, which can vary from one pad to an other. A relative calibration of each pad could be achieved by analysing the signal amplitude induced by an $\alpha$ particle traversing the detector. Indeed, since the Bragg peak is located at distances up to 4 times the detector length (depending on the gas pressure), the energy deposited (and therefore the number of primary electrons) on each pad is about the same, provided that the signal is not spread on several pads of the same row. This allows for the normalization of signal amplitudes. During the experiment we found very similar gains for all the pads (Fig. 2c).

- Evaluate the lowest neutron energy. Proton traces at neutron energies as low as $300 \mathrm{keV}$ have been obtained during the test. This fixes the lowest neutron energy at which the detector can be used at $300 \mathrm{keV}$. However tests at lower energies should be performed. Examples of the obtained proton trajectories are shown in panels $\mathrm{e}$ and $\mathrm{f}$.

Moreover, 6 days neutron irradiation of the electronic cards, which are placed very close to the detector (and therefore to the neutron source), did not affect the response of the electronics.

Two main issues were encountered during the test. First of all, the presence of residual oxygen in the chamber, as well as fluctuations in the gas pressure, induce sparks between the anode and the cathode. While a better purity of the gas with respect to $\mathrm{O}$ contamination could help, a possible solution under study consists in using a gas mixture.

Moreover, a charge accumulation on the insulator pieces under beam irradiation was observed. This induces a progressive reduction of the signal amplitude on the pads closest to the insulator pieces (i.e. on the first row of pads). The effect of the charge accumulation is shown in Fig. 3, where the signal amplitude of the first row of pads is plotted as a function of the irradiation time. The electrostatic charge is partially removed and one third of the signal amplitude recovered by connecting the collimator to the cathode and grounding it. The possibilities of adding a field cage, as well as using a less compact geometry to minimize the field distortions are under study. tested.

Finally the overall efficiency of the detector is to be

\section{Conclusions}

Neutron flux measurements via the elastic scattering $\mathrm{H}(\mathrm{n}, \mathrm{p})$ reaction is challenging for neutron energies below $1 \mathrm{MeV} . \gamma$-rays produced by the neutron production target generate electrons which induce a strong background in silicon detectors, preventing an accurate determination of the number of recoil protons. A new gaseous recoilproton detector has being designed and tested at the CENBG. Its low electron sensitivity is suited for neutron energies down to $300 \mathrm{keV}$ in a $\gamma \mathrm{s}$ and electrons dense environment, and a $\Delta \mathrm{E}-\mathrm{E}$ coincidence, coupled to a rough trajectory reconstruction, allow the discrimination of direct and scattered neutrons. Solutions are under study to avoid the effects of charge accumulation on insulator pieces occurring under beam irradiation. The main differences with respect to the well-established $\mathrm{H}$-filled cylindrical proportional counter developed by Bennett's et al. are discussed.

\section{References}

[1] G. Aliberti, G. Palmiotti, M. Salvatores, T. Kim, T. Taiwo, M. Anitescu, I. Kodeli, E. Sartori, J. Bosq, J. Tommasi, Annals of Nuclear Energy 33, 700 (2006)

[2] M. Chadwick, M. Herman, P. Oblozinsky, M. Dunn, Y. Danon, A. Kahler, D. Smith, B. Pritychenko, G. Arbanas, R. Arcilla et al., Nuclear Data Sheets 112, 2887 (2011)

[3] R. Rachamin, C. Wemple, E. Fridman, Annals of Nuclear Energy 55, 194 (2013)

[4] NEA, Nuclear Data High Priority Request List (2016), http://www.nea.fr/html/dbdata/hprl

[5] E.F. Bennett, Review of Scientific Instruments 33, 1153 (1962)

[6] J. Hassard, J. Liu, R. Mongkolnavin, D. Colling, Nuclear Instruments and Methods in Physics Research Section A: Accelerators, Spectrometers, Detectors and Associated Equipment 416, 539 (1998)

[7] K. Asai, N. Naoi, T. Iguchi, K. Watanabe, J. Kawarabayashi, T. Nishitani, Journal of Nuclear Science and Technology 43, 320 (2006)

[8] R. Babut, V. Gressier, Journal of Instrumentation 2, P01005 (2007)

[9] Y. Watanabe, Y. Naitou, S. Hirayama, M. Hayashi, A. Prokofiev, A. Hjalmarsson, S. Pomp, P. Andersson, R. Bevilacqua, C. Gustavsson et al., J. Korean Phys.Soc. 59, 1439 (2011)

[10] G. Kessedjian, G. Barreau, M. Aïche, B. Jurado, A. Bidaud, S. Czajkowski, D. Dassié, B. Haas, L. Mathieu, L. Tassan-Got et al., Phys. Rev. C 85, 044613 (2012)

[11] R. Beyer, E. Grosse, K. Heidel, J. Hutsch, A. Junghans, J. Klug, D. Légrády, R. Nolte, S. Röttger, 
M. Sobiella et al., Nuclear Instruments and Methods in Physics Research Section A: Accelerators, Spectrometers, Detectors and Associated Equipment 575, 449 (2007)

[12] A. Donzella, M. Barbui, F. Bocci, G. Bonomi, M. Cinausero, D. Fabris, A. Fontana, E. Giroletti, M. Lunardon, S. Moretto et al., Nuclear Instruments and Methods in Physics Research Section A: Accelerators, Spectrometers, Detectors and Associated Equipment 613, 58 (2010)

[13] M. Kovash, B. Daub, J. French, V. Henzl, K. Shoniyozov, J. Matthews, Z. Miller, H. Yang, in Advancements in Nuclear Instrumentation Measurement Methods and their Applications (ANIMMA), 2011 2nd International Conference (2011), pp. 1-3
[14] J. Taforeau, I. Schaefer, S. Higueret, D. Husson, L. Lebreton, Progress in Nuclear Science and Technology 4, 675 (2014)

[15] C. Cazzaniga, M. Rebai, M. Tardocchi, G. Croci, M. Nocente, S. Ansell, C.D. Frost, G. Gorini, 2015 (2015)

[16] P. Marini, L. Mathieu, L. Acosta, M. Aïche, S. Czajkowski, B. Jurado, I. Tsekhanovich, Nuclear Instruments and Methods in Physics Research Section A: Accelerators, Spectrometers, Detectors and Associated Equipment 841, 56 (2017)

[17] E. Pollacco, S. Anvar, H. Baba, P. Baron, D. Bazin, C. Belkhiria, B. Blank, J. Chavas, P. Chomaz, E. Delagnes et al., Physics Procedia 37, 1799 (2012) 